\title{
Potentiel de séquestration du carbone par les espaces verts aménagés urbains de la ville de Bobo-Dioulasso au Burkina Faso.
}

\author{
Alain P.K. GOMGNIMBOU1*, Wendsom Osée OUEDRAOGO2,3, Abdramane SANON1,4, Madjelia \\ KONE1, Daniel ILBOUDO 3 , Hassan B. NACRO4 \\ ${ }^{1}$ Centre National de la recherche Scientifique et Technologique/Institut de l'Environnement et de Recherches \\ Agricoles (INERA), Station de Farako-bâ, Bobo-Dioulasso (Burkina Faso) \\ ${ }^{2}$ Ecole Nationale des Eaux et Forêt (ENEF), Bobo Dioulasso, Burkina Faso, \\ 3Ministère de l'Environnement, de l'Economie Verte et du Changement Climatique (MEEVCC) \\ ${ }^{4}$ Université Nazi Boni (UNB)/Laboratoire d'étude et de recherche sur la fertilité des sols (LERF), de Bobo-Dioulasso, \\ Burkina Faso. \\ *Auteur pour la correspondance : Téléphone : +226 70 286633, E-mail : gpkalain@yahoo.fr \\ Original submitted in on $9^{\text {th }}$ September 2019. Published online at www.m.elewa.org/journals/ on $31^{\text {st }}$ December 2019 \\ https://doi.org/10.35759/JABs.v144.2
}

\section{RESUME}

Contexte et objectifs : Les espaces végétalisés des villes participent à l'épuration de l'air, à l'atténuation du changement climatique et à l'amélioration du cadre de vie. Toutefois, ces services écosystémiques sont peu connus des citadins, et rarement pris en compte dans les études. L'objectif de la présente étude est d'évaluer le potentiel de séquestration du carbone par les espaces verts dans la ville de Bobo Dioulasso.

Méthodologie et résultats : Afin d'évaluer leur contribution à l'atténuation du changement climatique, une étude d'estimation du stock de carbone dans les espaces verts de bobo a été menée. Le stock de carbone de la végétation ligneuse de 32 espaces verts aménagés a été estimé par la méthode non destructive en utilisant des équations allométriques. La végétation ligneuse est composée de 42 espèces, réparties dans 22 familles dont la plus représentée est celle des Fabaceae. Les quantités de carbone contenues dans la biomasse appartiennent ont varié de 3,22 tC/ha et 356,98 tC/ha en fonction des espaces. Le volume total de carbone séquestré a été estimé à 203,09 tonnes.

Conclusion et applicabilité des résultats : Le potentiel actuel de séquestration du carbone par les espaces verts est faible mais, peut être amélioré. Pour ce faire, la préservation du potentiel ligneux des espaces verts ainsi que la réalisation de plantations pourraient contribuer à accroitre leur stock de carbone.

Mots clés : biomasse, stock de carbone, changement climatique, espace vert, Bobo-Dioulasso, Burkina Faso. 


\section{ABSTRACT}

Contribution of carbon potential sequestration estimation by urban green spaces: case of the city of Bobo-Dioulasso in Burkina Faso

Context and objectives: The green spaces of cities contribute to the purification of the air, the mitigation of climate change and the improvement of the living environment. However, these ecosystem services are little known to urban populations, and rarely taken into account in studies.

The objective of this study is to evaluate a potential carbon stock by green spaces in the city of Bobo Dioulasso.

Methodology and results: In order to estimate their contribution in the mitigation of climate change, a work based on carbon stock was implemented. Not destructive method was employed and Allometric equations developed were used to evaluate the carbon store. The woody vegetation is composed of 42 species, divided into 22 families. The most represented being the Fabaceae. The amounts of carbon contained in the biomass are contained in the range $3.22 \mathrm{tC} / \mathrm{ha}$ and $356.98 \mathrm{tC} / \mathrm{ha}$. The total amount of sequestered carbon is estimated at 203.09 tons.

Conclusion and application of results: The potential carbon stock by green spaces is low but can be increased. The preservation of the woody potential of green spaces as well as the realization of plantations could contribute to increase their carbon stock.

Keywords: biomass, carbon stockage, climate change, green space, Bobo-Dioulasso, Burkina Faso.

\section{INTRODUCTION}

Les villes abritent de nos jours, un nombre relativement élevé de formations végétales qui sont soit plantées ou naturelles (Kouadio et al., 2016). Ces espaces végétalisés sont représentés par des parcs, des jardins publics ou privés, des plantations d'alignement de voiries, espaces vacants et remplissent des fonctions multiples pour les habitants (Polorigni et al., 2015 ; Ali-Khodja, 2010). Ces formations connues sous le vocable de forêts urbaines (Nowak et al., 2006) ou encore d'espaces verts urbains (Jo, 2002), offrent une multiplicité de services notamment les services écosystémiques (Tavin et Leseur, 2016, AliKhodja, 2010). Selon ces auteurs, il s'agit de la séquestration du carbone, la régulation thermique, les services de support de la biodiversité, de purification des polluants atmosphériques. Ces formations végétales urbaines servent également d'espaces de loisirs. Ils peuvent aider à atténuer les effets du changement climatique, par la fixation du carbone via la photosynthèse dans la biomasse ou dans le sol (Nowak et al., 2006). Selon le Code de l'urbanisme et de la construction (2006) du Burkina Faso, chaque projet de lotissement doit prévoir une zone qui sera spécifiquement dédiée à la végétation afin de garantir un cadre de vie agréable et un espace récréatif aux futurs habitants. Malheureusement ces espaces, font l'objet de diverses pressions à savoir les constructions de bâties du fait d'une faible connaissance des services écosystémiques qu'ils offrent, notamment l'immobilisation du carbone. Dans la ville de Bobo-Dioulasso, comme dans d'autres villes du pays, la forte demande foncière fait que la priorité est donnée à l'habitat et aux infrastructures au détriment des zones à vocation d'espaces verts récréatifs (Deronzier, 2017). En outre, la pollution urbaine prend de plus en plus de l'ampleur et il est donc indispensable de penser sur des moyens et des stratégies d'atténuation qui peuvent contribuer à la séquestration des gaz à effet de serre par les écosystèmes urbains, notamment les espaces verts aménagés. L'objectif général de cette étude est de contribuer à une meilleure connaissance des services écologiques que remplissent les espaces verts aménagés de la ville de Bobo Dioulasso dans un contexte de changement climatique. De façon spécifique, il s'agit d'évaluer la richesse floristique des espaces verts aménagés (EVA) et le potentiel de séquestration de carbone des EVA. 


\section{MATERIEL ET METHODES}

Présentation de la zone d'étude : La présente étude a été réalisée, en 2017, à Bobo-Dioulasso au Burkina Faso et qui s'étend sur 27000 ha (Deronzier, 2017). Les coordonnées géographiques de cette ville sont les suivantes : latitude $11^{\circ} 10^{\prime} 37$ Nord et longitude $4^{\circ} 17^{\prime}$ 52 Ouest. Elle est le chef-lieu de la province du Houet et de la région des Hauts-Bassins, qui se situe dans le sud-ouest du pays (Figure 1). Au recensement général de la population en 2006, la ville comptait 489967 habitants contre 813610 habitants en 2012 (INSD, 2016). Située dans le domaine sud Soudanien selon les travaux de Fontès et Guinko (1995), la zone d'étude est comprise entre les isohyètes de $900 \mathrm{~mm}$ et $1200 \mathrm{~mm}$. Elle est également caractérisée par l'alternance de deux saisons. La saison pluvieuse qui s'étend de mai à octobre et une saison sèche qui s'étend de novembre à avril avec toutefois quelques millimètres d'eau de pluie enregistrés en mars et avril. Sur les dix dernières années (2007-2017), la pluviosité moyenne annuelle enregistrée est estimée à 1045,27 $\pm 144,97 \mathrm{~mm}$ et la température moyenne annuelle est de $28,03 \pm 0,18{ }^{\circ} \mathrm{C}$ (llboudo, 2018). Dans la région de Bobo-Dioulasso, on rencontre des sols ferralitiques moyennement profonds, et remaniés sur des matériaux argilo-sableux issus de grès selon les résultats des travaux du Bureau National des Sols du Burkina Faso (BUNASOLS, 1985).

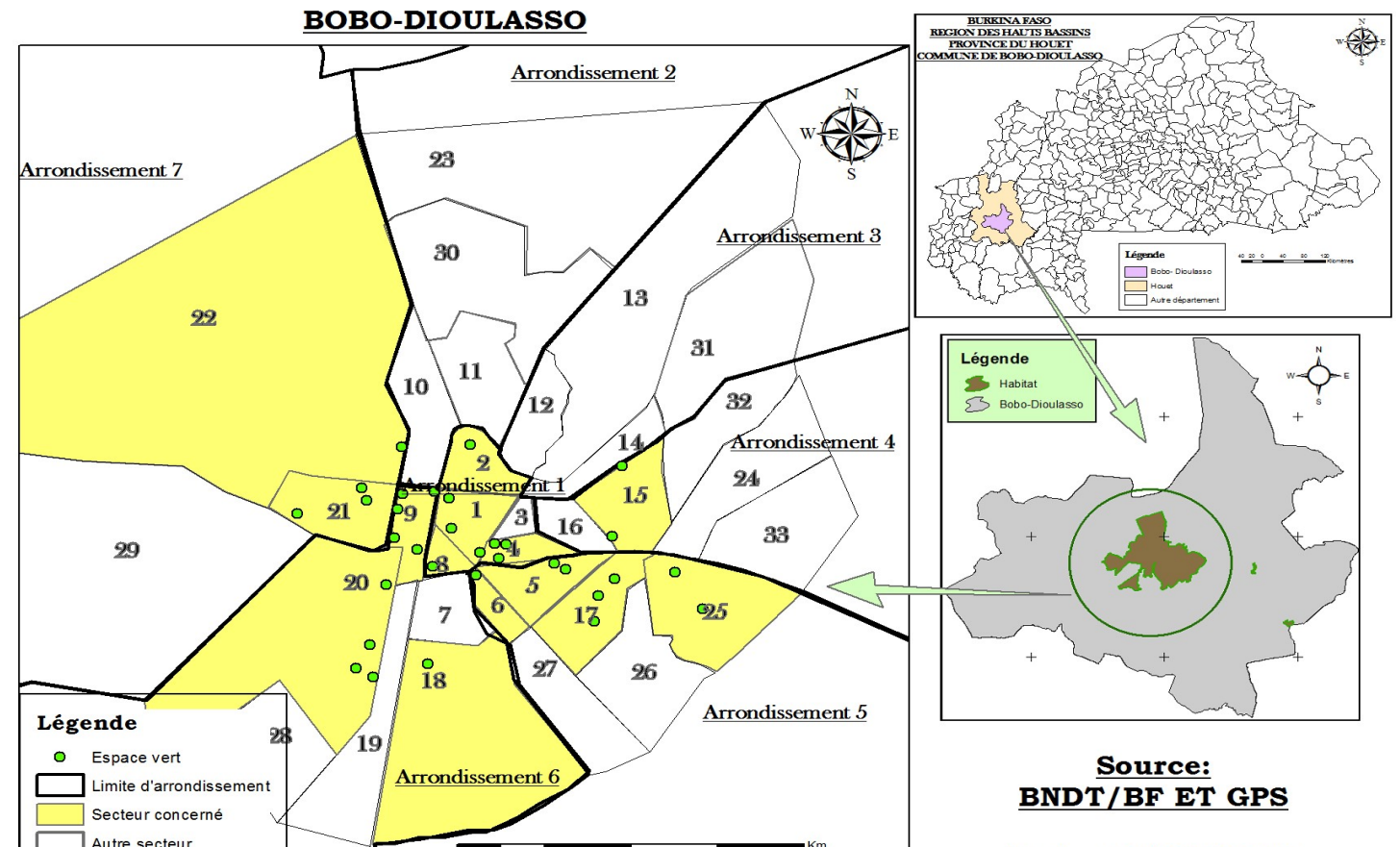

Figure 1 : Carte de localisation de la zone d'étude

Date: $16 / 08 / 2019$

\section{Méthodologie de l'étude}

Échantillonnage des espaces verts: L'exploitation des résultats des travaux de cartographie des espaces verts de la ville de Bobo-Dioulasso, réalisés par Traoré (2016) ainsi que le schéma d'aménagement urbain. Sur la base de ces données, tous les espaces verts aménagés qui existent ont été répertoriés pour la collecte des informations. Ains, c'est au total trentedeux (32) espaces verts aménagés qui ont permis de faire le diagnostic. Ils se répartissent dans cinq (05) communes d'arrondissement sur les sept (07) que compte la ville de Bobo-Dioulasso.
Collecte des données floristiques: Elle a été faite en faisant un inventaire intégral de l'ensemble des espèces trouvées dans chacun des espaces verts vu que les superficies sont réduites. La diversité floristique et structurale de la végétation ligneuse des EVA a été appréciée à partir de la distribution des tiges dans les classes de diamètre (Chevalier et al., 2010). A l'aide d'un ruban métrique, des mesures de la circonférence ont été effectuées à $1,30 \mathrm{~m}$ au-dessus du sol $\left(\mathrm{C}_{1,30 \mathrm{~m}}\right)$. Elle est ensuite convertie en diamètre à hauteur de poitrine $(\mathrm{DBH})$ par la relation : 
$D B M=C 1,30 / 3,14$ (1)

Seuls les individus dont le diamètre est supérieur ou égale à $3 \mathrm{~cm}$ ont été retenus.

Estimation de la biomasse et du stock de carbone séquestré: Les méthodes directes d'évaluation de la biomasse ligneuse permettent d'atteindre un niveau de précision élevé mais présentent des coûts considérables par rapport aux méthodes indirectes (Valentini, 2007). Dans le cadre de cette étude, les quantités de biomasse ont été déterminées à partir des équations allométriques générales des arbres urbains ou Urban General Equations (Aguaron et McPherson, 2012), qui ont la particularité d'aboutir à la biomasse totale (aérienne et racinaire) :

\section{Bĩom $(t)($ Arbres urbains $)=0,161555 x D B H^{2,310647}$}

où Biom (t) désigne la biomasse totale (aérienne et racinaire) exprimée en $\mathrm{kg}$ et $\mathrm{DBH}$ désigne le diamètre de la plante à hauteur de poitrine exprimé en centimètre $(\mathrm{cm})$.Ces modèles allométriques ne sont pas valables pour l'évaluation de la biomasse des palmiers. Pour ces derniers (Palmier à huile, rônier et cocotier), l'équation est celle développée par Brown (1997) pour les forêts tropicales sèches :

$$
B a=\exp (-2,134+2,53 \ln (D B H))(3)
$$

\section{RESULTATS}

Composition floristique des espaces verts aménagés : Les résultats de l'inventaire floristique ont donné au total 762 arbres et 2180 arbustes. La végétation ligneuse des EVA se compose de 42 espèces, réparties en 22 familles. La classification des familles par ordre décroissant de représentativité (figure où $\mathrm{Ba}=$ biomasse en $\mathrm{kg}, \mathrm{ln}=$ logarithme népérien et $\mathrm{DBH}=$ conversion du diamètre de la plante mesuré à $1,30 \mathrm{~m}$ au-dessus du sol, exprimé en $\mathrm{cm}$.

Pour aboutir au stock de carbone (tC/ha), la quantité de biomasse (t/ha) a été multipliée par le facteur de conversion qui est 0,5 suivant les recommandations du GIEC (2003), selon la formule :

Stock de Carbone $=$ Quantité de biomasse aérienne (AGB) x 0,5 (Facteur de conversion).

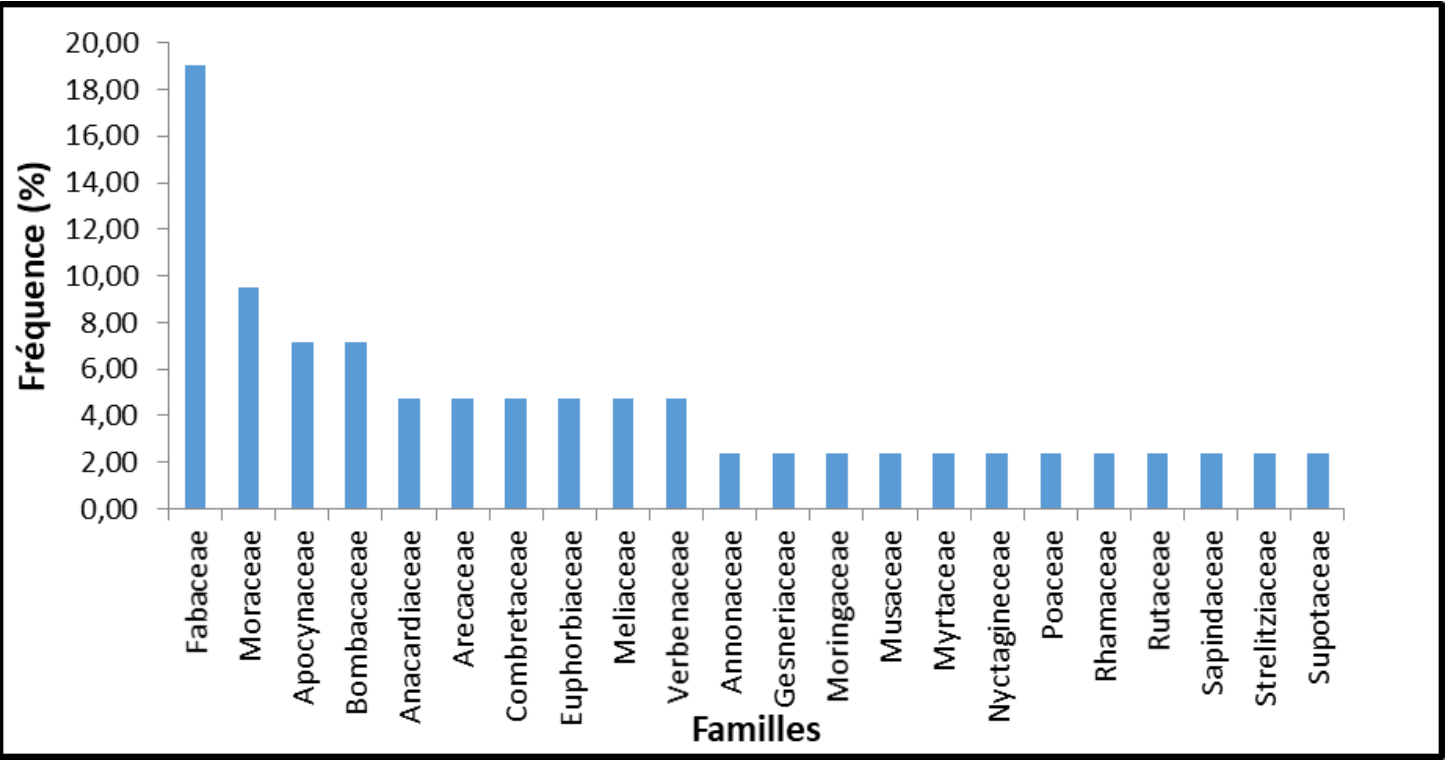

Figure 2 : Composition floristique des EVA étudiés.

2) indique que les Fabaceae $(19,04 \%)$ sont les plus nombreuses suivies des Moraceae (9,52\%). Les familles des Annonaceae, Apocynaceae, Moringaceae, Myrtaceae, Rhamaceae, Rutaceae, Sapindaceae, Poaceae, Musaceae, Nyctagineceae, Strelitziaceae, Sapotaceae sont les moins représentées (2,38\%). 
Biomasse et quantités de carbone séquestré : Les paramètres structuraux, le volume de biomasse, de carbone sont donnés dans le tableau 1. II ressort que les superficies des EVA varient entre 0,02 à 1,2 ha. La densité la plus faible est de 10,4 arbres/ha retrouvée dans l'arrondissement 1, tandis que la plus élevée $(4717,83$ arbres/ha) a été rencontrée dans l'arrondissement 4 (tableau 1). Les quantités de biomasse diffèrent considérablement d'un EVA à un autre. Elles varient de 6,44 t/ha à 713,97 t/ha pour un stock de carbone correspondant oscillant entre 3,22 tC/ha et 356,98 tC/ha (tableau 1). L'arrondissement 6 a enregistré le cumul de biomasse le plus élevé soit 1 159,35 t/ha.

Tableau 1. Paramètres structuraux, biomasse et stock de carbone des espaces verts de la ville de Bobo-Dioulasso

\begin{tabular}{|c|c|c|c|c|c|}
\hline Commune & Espace vert & Superficie (ha) & $\begin{array}{l}\text { Densité } \\
\text { (arbres/ha) }\end{array}$ & AGB (t/ha) & Carbone (tC/ha) \\
\hline \multirow{9}{*}{$\begin{array}{l}\text { Arrondis } \\
\text { sement } 1\end{array}$} & Jardin Samora & 0,17 & 970,37 & 58,13 & 29,06 \\
\hline & Le café des amis & 0,02 & 2636,36 & 218,13 & 109,06 \\
\hline & Jardin de la renaissance & 0,10 & 1900,00 & 420,54 & 210,27 \\
\hline & Jardin de la LONAB & 0,04 & 1773,05 & 81,11 & 40,55 \\
\hline & Jardin Kaboré & 0,27 & 688,89 & 117,97 & 58,98 \\
\hline & Monument des Martyrs & 0,19 & 246,41 & 27,91 & 13,95 \\
\hline & Jardin $25^{\text {ème }}$ heure & 0,20 & 245,00 & 19,55 & 9,77 \\
\hline & Maquis Free love & 0,07 & 742,86 & 95,10 & 47,55 \\
\hline & Sous total 1 & - & - & 1038,23 & 519,19 \\
\hline \multirow{3}{*}{$\begin{array}{l}\text { Arrondis } \\
\text { sement } 4\end{array}$} & Maquis le freedom & 0,20 & 365,00 & 79,50 & 39,75 \\
\hline & Buvette Zoodia & 0,04 & 4717,83 & 85,49 & 42,75 \\
\hline & Sous total 2 & & & 164,99 & 82,5 \\
\hline \multirow{9}{*}{$\begin{array}{l}\text { Arrondis- } \\
\text { sement } 5\end{array}$} & Jardin Zoodia & 0,91 & 51,86 & 26,77 & 13,39 \\
\hline & Carrefour du peuple & 0,80 & 87,50 & 9,28 & 4,64 \\
\hline & La cabane & 0,03 & 2920,00 & 376,73 & 188,37 \\
\hline & Maquis Soutra & 0,04 & 727,27 & 124,65 & 62,32 \\
\hline & Maquis le Rakiré-Wamdé & 0,26 & 1214,12 & 24,98 & 12,49 \\
\hline & Jardin la fontaine & 0,26 & 2578,00 & 39,97 & 19,98 \\
\hline & Jardin les trois manguiers & 0,25 & 778,09 & 97,65 & 48,83 \\
\hline & Maquis Déo-gracias & 0,12 & 900,00 & 6,44 & 3,22 \\
\hline & Sous total 3 & - & - & 666,47 & 353,24 \\
\hline \multirow{11}{*}{$\begin{array}{l}\text { Arrondis- } \\
\text { sement } 6\end{array}$} & Jardin Sidwaya & 1,20 & 93,33 & 26,22 & 13,11 \\
\hline & Jardin Idéal plus & 0,19 & 878,95 & 52,65 & 26,33 \\
\hline & Jardin Idéal annexe & 0,90 & 91,11 & 32,03 & 16,02 \\
\hline & Maquis ka-ya Wooto & 0,01 & 2372,88 & 713,97 & 356,98 \\
\hline & Buvette Nong-taaba & 0,03 & 1366,67 & 64,07 & 32,03 \\
\hline & Maquis le Colsa & 0,10 & 10,41 & 135,67 & 67,83 \\
\hline & Jardin Kossyam & 0,15 & 452,13 & 72,62 & 36,31 \\
\hline & Jardin de l'auberge Lafia & 0,45 & 272,49 & 22,33 & 11,17 \\
\hline & Jardin CSPS Lafiabougou & 0,25 & 439,87 & 11,66 & 5,83 \\
\hline & Maquis le trigone & 0,17 & 219,02 & 28,13 & 14,07 \\
\hline & Sous total 4 & - & - & 1159,35 & 579,68 \\
\hline \multirow{6}{*}{$\begin{array}{l}\text { Arrondis- } \\
\text { sement } 7\end{array}$} & Association Siraba & 0,39 & 407,63 & 89,26 & 44,63 \\
\hline & Claire Amitié & 0,29 & 129,83 & 89,67 & 44,83 \\
\hline & Maquis de l'antenne & 0,14 & 71,84 & 32,45 & 16,22 \\
\hline & Maquis détente & 0,12 & 869,94 & 31,92 & 15,96 \\
\hline & Sous total 5 & - & - & 243,3 & 121,64 \\
\hline & TOTAL & - & - & 3272,34 & 1656,25 \\
\hline
\end{tabular}

AGB=Quantité de biomasse aérienne 


\section{DISCUSSION}

Diversité végétale: Les résultats obtenus sur la composition et la richesse floristique, montrent que la famille des Fabacae est la plus représentée. Cela pourrait s'expliquer par la forte représentativité des espèces locales. Elles représentent, en effet, $65,75 \%$ des arbres inventoriés et $19,95 \%$ des arbustes inventoriés. Ce résultat se rapproche des $63,8 \%$ d'espèces locales et $36,2 \%$ d'espèces exotiques inventoriées par Monssou et al. (2016) dans le Jardin Botanique de Bingerville en Côte d'Ivoire. Les espèces exotiques sont relativement moins représentées, avec $34,25 \%$ des arbres inventoriés et $80,05 \%$ des arbustes inventoriés. On les retrouve principalement suivant l'ordre de représentativité dans la deuxième famille, les Moraceae, dont la proportion est de $9,52 \%$. En termes de nombre d'espèces, la relative importance des Moraceae serait liée à la forte contribution du genre Ficus à l'embellissement des jardins publics. Les individus appartenant à l'espèce, Ficus benjamina, $F$. loricata notamment, taillés, améliorent significativement l'aspect décoratif des dits jardins. D'autres par contre sont reconnus pour être des sujets ombrageux, et plantés dans les espaces de loisirs à Bobo-Dioulasso. Le nombre d'espèces varie d'un EVA à un autre. Le jardin de la Renaissance qui compte 19 espèces est le plus diversifié des EVA étudiés. Cependant, le maquis Antenne du Secteur 21 offre la plus faible richesse floristique avec seulement 2 espèces. L'insuffisance dans l'entretien des plantes ainsi que l'absence d'une politique de gestion des espaces verts seraient à l'origine de cette faible diversité floristique. Cette situation a déjà été évoquée par d'autres auteurs (Polorigni et al., 2015 ; Ali-Khodja, 2010). Les résultats sur la densité des arbres enregistrée au cours de l'étude indiquent que celle-ci varie d'un espace vert à un autre. De manière générale, on constate que le nombre d'arbres/arbustes à l'hectare est relativement important. Un nombre de plus 2000 arbres/ha a été relevé dans certains EVA. Cela s'explique par le fait que ces jardins ont été aménagés sur d'anciens sites reboisés afin de préserver le potentiel ligneux lors des opérations de lotissement. Ce résultat est largement supérieur à la densité des tiges du Jardin Botanique de Bingervillle, qui varie de 88 à 460 arbres/ha (Monssou et al., 2016). II est également plus important aux $130,76 \pm 147,95$ tiges/ha relevées sur les avenues ainsi qu'aux 37,35 $\pm 20,70$ tiges/ha trouvées au niveau des boulevards des communes du Plateau et de Coccody par Kouadio et al. (2016). La prise en compte des individus de petits diamètres $(\mathrm{DBH} \geq 3 \mathrm{~cm})$ de même que la présence de haies vives, pourraient expliquer les valeurs, parfois, très élevées de la densité. Au niveau desdites haies vives à base de Bohinia rufescens, Cascabela thevetia, Durenta rupens ou de Bougainvillia spectabilis, les écartements entre plants sont faibles, souvent, inférieurs à un (01) m. Ainsi, aboutit-t-on à une forte densité ligneuse au niveau ce type d'aménagement de végétaux.

Biomasse et potentiel de stockage du carbone : Les quantités de biomasse (variant de 6,44 t/ha à 713,97 t/ha) ainsi que le stock de carbone $(3,22 \mathrm{tC} / \mathrm{ha}$ et $356,98 \mathrm{tC} /$ ha par EVA), diffèrent considérablement d'un EVA à un autre. Ce résultat est largement plus important par rapport à ceux trouvés par Monssou et al. (2016) dans le Jardin Botanique de Bingerville, où les valeurs de biomasse varient de 21 à 381,4 tha. II est également supérieur aux valeurs de biomasse des jardins de la Commune du Plateau d'Abidjan qui varient de 19,25 à 181,60 tha selon les résultats des travaux de Vroh et al. (2014). Au niveau des avenues et des boulevards des communes du Plateau et de Coccody, Kouadio et al. (2016) ont trouvé respectivement 562,33 $\pm 819,39$ t/ha et $266,09 \pm 363,48$ tha. Ces valeurs de biomasse sont peu élevées par rapport aux 6,44 à 713,97 t/ha enregistrées au niveau des EVA de la ville de Bobo-Dioulasso. Ces différences seraient liées à la démarche méthodologique de collecte des données. Alors que notre étude a pris en compte les sujets de petits diamètres (DBH $\geq 3 \mathrm{~cm}$ ), les autres auteurs précédemment évoqués, n'ont considéré que les individus dont le diamètre est supérieur ou égal à 10 $\mathrm{cm}$. Considérant tous les EVA de la ville de BoboDioulasso, le maquis Ka-ya-Wooto renferme le plus important stock de carbone qui est de 356,98 tC/ha. La densité élevée (2372,88 tiges/ha), l'important pool d'individus de gros diamètres de cet EVA pourrait justifier la supériorité de son volume de carbone en comparaison aux autres. Comme l'ont relevé Amougou et al. (2016), les stocks de carbone dépendent de plusieurs paramètres qui sont entre autres la qualité et la quantité des arbres. De même, la forte contribution des sujets de gros diamètres aux stocks totaux de biomasse a été démontré par d'autres études (Joosten et al., 2004; Mbow, 2009). 


\section{CONCLUSION}

Ce travail avait pour objectif d'étudier la composition floristique de la végétation ligneuse des EVA de la ville de Bobo-Dioulasso. II s'agissait également d'évaluer la biomasse afin d'en déduire les quantités de carbone séquestré. On retiendra de l'étude que les espaces verts de la ville de Bobo-Dioulasso ont une richesse floristique faiblement diversifiée. Le potentiel de séquestration varie considérablement d'un espace vert à un autre. Toutefois, près de $68 \%$ des espaces ont un potentiel relativement faible de stockage de carbone du fait de leur dégradation. L'étude a également révélé le faible niveau d'aménagements végétaux des espaces verts. Les autorités municipales devraient, par conséquent, sensibiliser les gestionnaires ou occupants de ces espaces en matière de création et de préservation des plantations de ces milieux. Ainsi, la nécessité de mettre en œuvre de meilleures stratégies de gestion durable de ces espaces s'impose.

\section{REMERCIEMENTS}

Les auteurs remercient le Cercle d'Assistance et de Conseils en Environnement (CACE) pour l'appui financier et technique apporté à la réalisation de la présente étude.

\section{REFERENCES BIBLIOGRAPHIQUES}

Ali-Khodja, A. 2010. Aménagement urbain : la problématique de l'espace vert public dans la ville de Constantine. Science et technologie 32: $9-18$

Aguaron, E. and McPherson, E. G. 2012. Comparison of methods for estimating carbon dioxide storage by Sacramento's Urban Forest. Ecosystems and Social Dynamics Program, USDA Forest Service, 1731 Research Park Dr, Davis, CA 95618, USA, 43-71.

Amougou, J. A., Bembong Ebokona, L. D., Batha, R. A. S., Mala, A. W. and H. Ngono. 2016. Estimation du stock de carbone dans deux unités de terre en zone de savane du Cameroun. Revue de Géographie, d'Aménagement Régional et du Développement des Suds, Regardsuds 2:2845.

Brown, S. 1997. Estimating biomass and biomass change of tropical forest: A Primer. Forest Ressources Assement Publication; Forestry Paper 134, FAO, $55 \mathrm{p}$.

BUNASOLS (Bureau National des Sols). 1985. Etude pédologique de la forêt classée de Dindéresso, province du Houet, échelle 1/120000. Rapport du Bureau National des Sols; Ouagadougou, Burkina Faso.

Chevalier, R., Gautier, G., Arcahux, F. 2010. Relevés floristiques pour le suivi de la biodiversité végétale des écosystèmes forestiers : éléments de réflexion pour les bons choix. Revue des Forêts Françaises 72 (2):141-155.

Deronzier, M. 2017. Articulation ville/nature en Afrique de l'Ouest. Systèmes de gestion et diversité des rapports liés à la biodiversité végétale dans la ville de Bobo-Dioulasso (Burkina Faso). Mémoire de master, AgroParisTech / Université Paris-Saclay, Université Paris Sorbonne, $104 \mathrm{p}$.

Fontès, J. et S. Guinko. 1995. Carte de végétation et de l'occupation d'un sol du Burkina Faso. Notice Explicative. Ministère de La Coopération Française, Projet Campus (8813101), Toulouse, $67 \mathrm{p}$.

GIEC. 2003. Recommandations du GIEC en matière de bonnes pratiques pour le secteur de l'utilisation des terres, changements d'affectation des terres et foresterie. IGES, Kanagawa, Japon, $25 \mathrm{p}$.

Ilboudo, D. 2018. Evaluation de la biomasse et du potentiel de séquestration de carbone par les petites formations forestières des savanes en zone soudanienne du Burkina Faso: cas du site de l'ENEF. Mémoire d'Ingénieur du Développement Rural, IDR/UNB, Burkina Faso, $62 \mathrm{p}$.

INSD. 2016. Annuaire statistique 2015. Ministère de l'économie, des Finances et du Développement, Institut National de la Statistique et de la Démographie (INSD), Burkina Faso, 397p.

Jo, H.-K. 2002. Impacts of urban greenspace on offsetting carbon emissions for middle Korea. Journal of Environmental Management 64:115-126.

Joosten, R., Schumacher, J., Wirth, C. and A., Schulte. 2004. Evaluating tree carbon predictions for beech (Fagus sylvatica L.) in Western Germany. Forest Ecology and Management 189: 87-96. 
Kouadio, Y. J. C., Vroh, B. T.A., Goné BI, Z. B., Adou Yao, C. Y. and K.E., N'Guessan. 2016. Évaluation de la diversité et estimation de la biomasse des arbres d'alignement des communes du Plateau et de Cocody (Abidjan Côte d'Ivoire). Journal of Applied Biosciences 97: 9141-9151.

Mbow, C. 2009. Potentiel et dynamique des stocks de carbone des savanes soudaniennes et soudano- guinéennes du Sénégal. Thèse de Doctorat d'Etat, UCAD, Dakar, Sénégal, 291 p.

Monssou, E. O., Vroh, B. T. A., Goné, Bl. Z. B., Adou, Yao. C. Y. et N'Guessan, K. E. (2016). Evaluation de la diversité et estimation de la biomasse aérienne des arbres du Jardin Botanique de Bingerville (District d'Abidjan, Côte d'Ivoire). European Scientific Journal 12 (6): 168-184.

Nowak, D. J., Hoehn, Robert, E., Daniel, E., Stevens, Jack, and C., Jeffrey. 2006. Assessing urban forest effects and values, Minneapolis' urban forest. Resour. Bull. NE-166. Newtown Square, PA: U.S. Department of Agriculture, Forest Service, Northeastern Research Station, $20 \mathrm{p}$.

Polorigni B., Radji R.A. and K., Kokou. 2015. Politique publique de gestion des espaces verts de la ville de Lomé au Togo. International Journal of Biological and Chemical Science 9 (4): 1888-1901

Tavin, A. and A., Leseur. 2016. Végétaliser la ville Pour quels bénéfices, avec quels financements, suivis et gouvernances des projets? L'apport d'exemples européens et nord-américains. I4CE, Institute for Climate Economics, $33 \mathrm{p}$.

Traoré, L. 2016. Évaluation des espaces verts dans la ville de Bobo-Dioulasso. Mémoire d'Inspecteur des Eaux et Forêts, ENEF de Bobo Dioulasso, Burkina Faso.

Valentini, S. G. 2007. Evaluation de la séquestration du carbone dans des plantations agroforestières et des jachères issues d'une agriculture migratoire dans les territoires autochtones de Talamanca, au Costa-Rica. Mémoire de master, Université Laval, Québec, Canada, 88 p.

Vroh, B. T. A., Tiebre, M. S. and K. E., N'Guessan. 2014. Diversité végétale urbaine et estimation du stock de carbone : cas de la commune du Plateau Abidjan, Côte d'Ivoire. Afrique Science 10 (3): 329-340. 